Electromagnetic, complex image model of a large area RF resonant antenna as inductive plasma source

This content has been downloaded from IOPscience. Please scroll down to see the full text. 2017 Plasma Sources Sci. Technol. 26035010

(http://iopscience.iop.org/0963-0252/26/3/035010)

View the table of contents for this issue, or go to the journal homepage for more

Download details:

IP Address: 128.178.126.249

This content was downloaded on 23/02/2017 at 11:49

Please note that terms and conditions apply. 


\title{
Electromagnetic, complex image model of a large area RF resonant antenna as inductive plasma source
}

\author{
Ph Guittienne ${ }^{1}$, R Jacquier ${ }^{2}$, A A Howling ${ }^{2}$ and I Furno ${ }^{2}$ \\ ${ }^{1}$ Helyssen, Route de la Louche 31, CH-1092 Belmont-sur-Lausanne, Switzerland \\ ${ }^{2}$ Ecole Polytechnique Fédérale de Lausanne (EPFL), Swiss Plasma Center, CH-1015 Lausanne, \\ Switzerland \\ E-mail: alan.howling@epfl.ch
}

Received 17 November 2016

Accepted for publication 17 January 2017

Published 22 February 2017

\begin{abstract}
A large area antenna generates a plasma by both inductive and capacitive coupling; it is an electromagnetically coupled plasma source. In this work, experiments on a large area planar RF antenna source are interpreted in terms of a multi-conductor transmission line coupled to the plasma. This electromagnetic treatment includes mutual inductive coupling using the complex image method, and capacitive matrix coupling between all elements of the resonant network and the plasma. The model reproduces antenna input impedance measurements, with and without plasma, on a $1.2 \times 1.2 \mathrm{~m}^{2}$ antenna used for large area plasma processing. Analytic expressions are given, and results are obtained by computation of the matrix solution. This method could be used to design planar inductive sources in general, by applying the termination impedances appropriate to each antenna type.
\end{abstract}

Keywords: inductively coupled plasma, complex image, multi-conductor transmission line, resonant antenna

(Some figures may appear in colour only in the online journal)

\section{Introduction}

Uniform plasma processing over large areas $\left(>1 \mathrm{~m}^{2}\right)$ is required for the industrial production of solar cells, flat panel displays, packaging, and surface treatment, etc. Inductively coupled plasma (ICP) reactors are standard in the semiconductor industry, and are being further developed for large area applications [1-5].

Electromagnetic (EM) effects such as standing wave nonuniformity can arise in ICP antenna sources [6-13] when the reactor size is non-negligible compared to the RF excitation wavelength, as well as in capacitively coupled (CCP) reactors [14-17]. Furthermore, capacitive coupling occurs in ICP reactors $[13,14,18]$, just as inductive coupling occurs in CCP reactors [15]. Hence the need for an electromagnetic model of large area ICP antenna sources: calculations of the antenna characteristics are necessary for source design and prediction of plasma properties, as well as for comprehension and interpretation of measurements. For example, the influence of plasma coupling on the antenna input impedance determines the power transfer efficiency [19] and the RF power matching conditions.

\subsection{Existing models of ICP sources}

The transformer model [1, 14, 20], using discrete (lumped) circuit elements to represent self-inductances and mutual inductance with the plasma, is often used to model solenoid and coil ICP sources, including spiral coils [21]. The inclusion of parasitic capacitive coupling improves the model $[14,22]$. For large area ICP sources, EM standing wave effects have previously been described by treating long coils as a single, lossy transmission line [6-9], where the transformer model was used to calculate the per-unit-length coupling to the plasma. ICP antennas were also treated as segments of an immersed transmission line [10]. These models do not consider capacitance and mutual inductance 
matrices between all the antenna elements and the plasma [7]. Mashima et al [23] used the moment method but plasma coupling was not taken into account. Numerical simulation can include EM effects via the wave field equations [11-13] although the solution requires longer computation.

In this paper, a large area ICP antenna array is modeled as a multi-conductor transmission line (MTL) [24]. The results of this EM model are compared with experiments performed on a $1.2 \times 1.2 \mathrm{~m}^{2}$ planar antenna which consists of an array of identical resonant circuit elements. When excited at one of its resonance frequencies, the RF resonant antenna develops very high currents within its structure, which can be used as an ICP source [19, 25-29]. Due to the spatially distributed resonant network, they are good candidates for large area plasma processing, with a particular advantage concerning impedance matching and power transfer efficiency because of their high and real input impedance. Previous models of small resonant network reactors successfully described antenna-plasma loading by accounting for lumpedelement mutual inductance between the antenna elements and the plasma; a mutual inductance matrix was calculated in terms of plasma properties using the complex image method [30, 31].

For the large area source, however, experiments show that plasma can be maintained even for an electrically floating antenna, which means that strong capacitive currents flow from the antenna via the plasma to ground. Modeling and ICP source design therefore also require the calculation of the associated capacitance matrix. The self-consistent calculation of antenna currents and voltages coupled by the mutual inductance matrix and the capacitance matrix is achieved here by considering the array of antenna legs as a MTL [24] coupled with the plasma. These considerations could also be relevant to other large area antenna reactor designs [1-3] such as the ladder antenna [23, 32-43], the serpentine antenna $[10,12,44]$, the U-type antenna [45-47] and the double comb-type antenna [48-50].

\subsection{Comparison of large area ICP sources}

External coil ICP reactors have the scale-up problem that the thick dielectric window, necessary to withstand atmospheric pressure over a large area, reduces the mutual inductance with the plasma [4-7, 11, 40, 51]. Internal coil ICP reactors can increase inductive coupling with the plasma and avoid the necessity of a thick dielectric window [52-55] by placing the antenna inside the vacuum chamber. Large area ICP sources are often an array of parallel linear legs [40]. The legs can be immersed in the plasma, with the legs individually isolated by a dielectric sleeve (serpentine [10, 12, 44], U-shape [45-47], comb-type [48, 49]), or directly exposed to the plasma (for example, versions of the ladder type [2, 36, 40]). Alternatively, the whole ICP antenna can be embedded in a dielectric, protected from the plasma by a thin window [22, 5659]. In this context, the large area resonant antenna in this work is an internal, embedded ICP source.

This paper is organized as follows: first, the large-area antenna experimental setup is described in section 2. A MTL model of the antenna is introduced in section 3 which uses a mutual partial inductance matrix and a capacitance matrix. The MTL model is compared with measurements of node voltages and antenna input impedance spectra without plasma in section 4. Plasma loading and uniformity measurements for the large antenna operating as an ICP source are interpreted using the MTL model in section 5 before concluding.

\section{Experimental setup}

A schematic of the experimental setup is shown in figure 1 . The antenna network is made up of 25 water-cooled tubular copper legs, length $120 \mathrm{~cm}$, external radius $0.4 \mathrm{~cm}$, with parallel axes $5 \mathrm{~cm}$ apart. The legs are connected at both ends by stringers consisting of $375 \mathrm{pF}$ ceramic capacitor assemblies with copper strip connectors $0.6 \mathrm{~cm}$ wide. The antenna assembly is set in a closed aluminium housing of internal dimensions $135 \times 135 \times 13 \mathrm{~cm}^{3}$. The leg axes are positioned $5 \mathrm{~cm}$ above the baseplate to limit eddy current power loss from inductive coupling with this plate. To avoid spurious plasma below and within the antenna network, the lower part of the reactor is filled with silica-alumina 85\%-porosity foam dielectric. This low-permittivity dielectric also minimizes capacitive coupling, and hence RF capacitive current, to the baseplate. The antenna surface is separated from the plasma by $0.3 \mathrm{~cm}$ thick glass; this thin dielectric window promotes strong mutual inductive coupling with the plasma, although the close proximity to the plasma means that capacitive coupling must also be taken into account. Any small gaps between the foam, glass and legs are filled with microsphere glass beads to prevent parasitic plasma below the glass. The plasma boundaries are the glass surface, the grounded metal top-plate $7 \mathrm{~cm}$ above the glass which defines the plasma gap, and the grounded metal sidewalls. The whole reactor was placed inside a vacuum vessel.

The RF input power is centrally connected along one side via a coaxial cable at node A13 in figure 1(b) whose ground shield connects to the baseplate. In this way, the RF current circulates only on the inside of the closed reactor which acts as a Faraday screen; this is important to suppress electric field leakage and parasitic plasma ignition in the space between the reactor and the vacuum vessel walls [2]. Inside the reactor, the antenna was either grounded to the baseplate via connections A10 and A16 (to preferentially excite mode $m=8$ [25]), or left unconnected for an electrically floating antenna.

The antenna impedance spectrum without plasma was measured with a network analyser, and the network node voltages $A n$ and $B n$ were measured with high impedance probes. The plasma-coupled network was electrically characterized by its input impedance and RF power using a current-voltage-phase probe $\left(\mathrm{Z}-\mathrm{Scan}^{\mathrm{TM}}\right.$ [60]) at the vacuum feedthrough between the matching box and the antenna. Plasma density profiles were measured using a uniform surface array of 100 negatively biased $(-30 \mathrm{~V})$ multiplexed probes flush with the top-plate, and a laterally traversing Langmuir probe. 
(a)

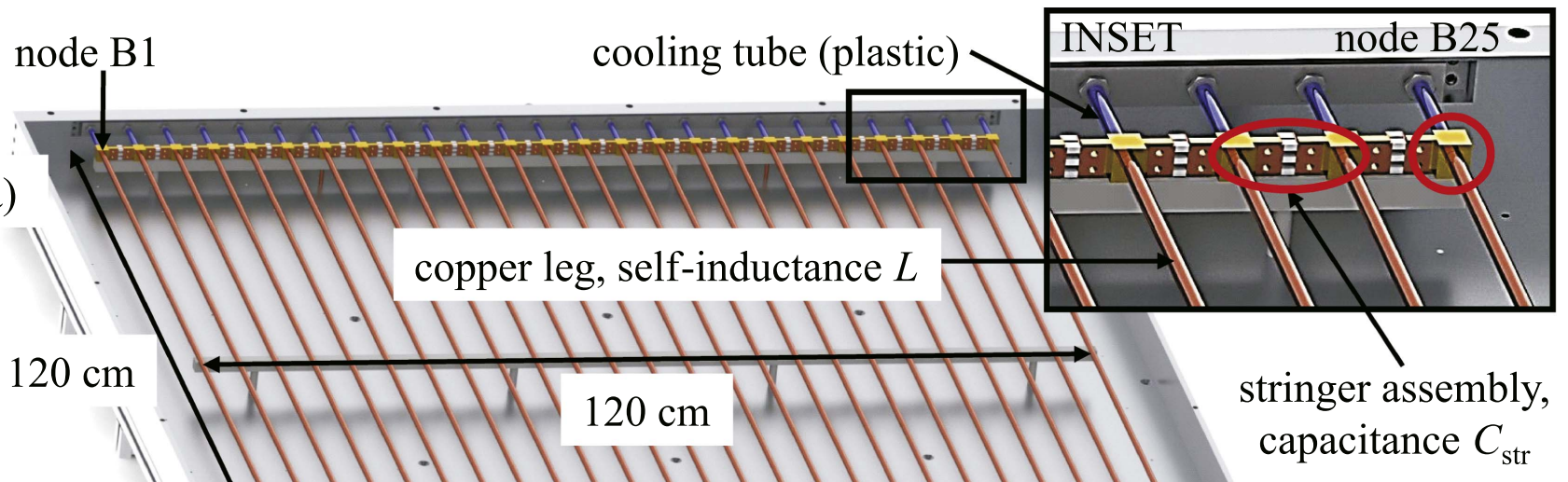

(b)

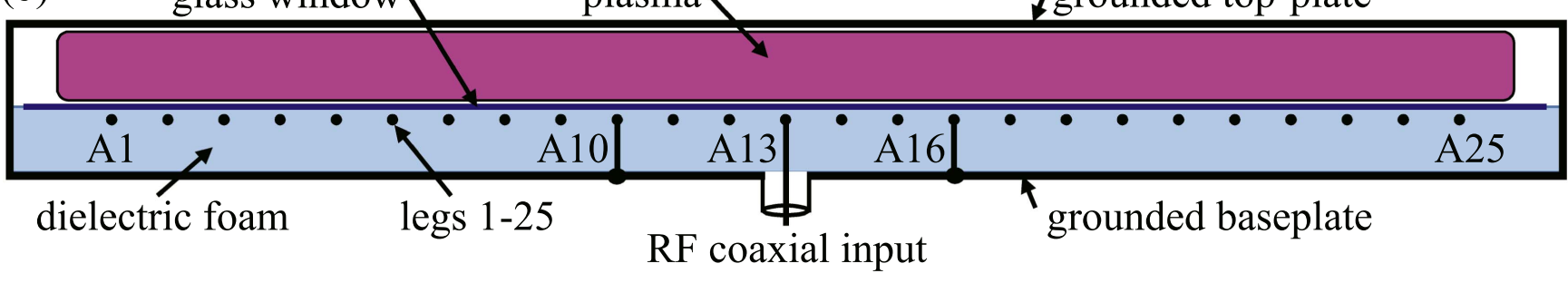

Figure 1. Scale drawings of the planar resonant antenna assembly. (a) Open top view of the empty antenna, with legs considered as inductances $L$ in a multi-conductor transmission line, joined at their ends by stringer capacitances $C_{\text {str }}$ shown in the inset. (b) End view of the closed reactor showing the antenna electrical connections: RF coaxial input at node A13; ground connections at A10 and A16.

The plasma parameters were $1.5-2 \mathrm{~Pa}$ argon pressure with 500-2000 $\mathrm{W}$ delivered power over a frequency range from 12 to $14 \mathrm{MHz}$, and $15 \mathrm{~kW}$ at $13.56 \mathrm{MHz}$.

\section{The multi-conductor transmission line (MTL) model for plasma-coupled antennas}

This section introduces the electromagnetic model which will be used to interpret the experimental results in sections 4 and 5. Reliable calculations of the antenna characteristics are necessary for source design and prediction of plasma properties, as well as for comprehension and interpretation of measurements. For example, the influence of plasma coupling on the antenna input impedance determines the choice of capacitors for a given operating frequency, the power transfer efficiency [19], and the RF power matching conditions.

\subsection{Equivalent circuit for antenna-plasma coupling}

In previous work on a small antenna [30, 31], a lumpedelement equivalent circuit gave a good representation of the experimental measurements because the antenna legs were very short $(0.2 \mathrm{~m})$ compared to the wavelength of the RF excitation $(22 \mathrm{~m}$ in vacuum at $13.56 \mathrm{MHz})$. Mutual inductance calculations were critically necessary in that work to accurately model the measured impedance spectra
$[30,31,61]$. However, the present antenna is six times longer, and experimental observations can no longer be accurately explained by the lumped-element mutual inductance model. For example, it was observed that plasma can be maintained with no antenna ground connection; the return current must all be flowing by capacitive coupling via the plasma to ground. This is incompatible with a purely inductive model.

As a rule of thumb in the EM treatment of large area (or very high frequency) sources, standing-wave non-uniformity generally occurs when the reactor size $L$ is longer than a tenth of the free-space wavelength of the RF excitation [15, 62], i.e. EM effects are expected for $f L>0.1 c$, where $f$ is the frequency of the RF excitation and $c$ is the speed of light in vacuum. The antenna stringers are much shorter than the legs and so a lumped-element approximation holds for these. Only standing waves in the long legs will be taken into account in the following calculations. The stringers enter the equations as termination impedances in appendix A.2.

The simultaneous capacitive and inductive coupling implies a transmission line treatment, where the multiple parallel leg conductors mean that the antenna can be described as a MTL [24] as shown in figure 2. Strictly speaking, the large antenna ICP generates a plasma which is simultaneously a CCP and an ICP; it would therefore be better described as an electromagnetically coupled plasma (EMCP) source. The MTL model will be developed here to calculate the input impedance of the antenna, and the distribution of currents and 


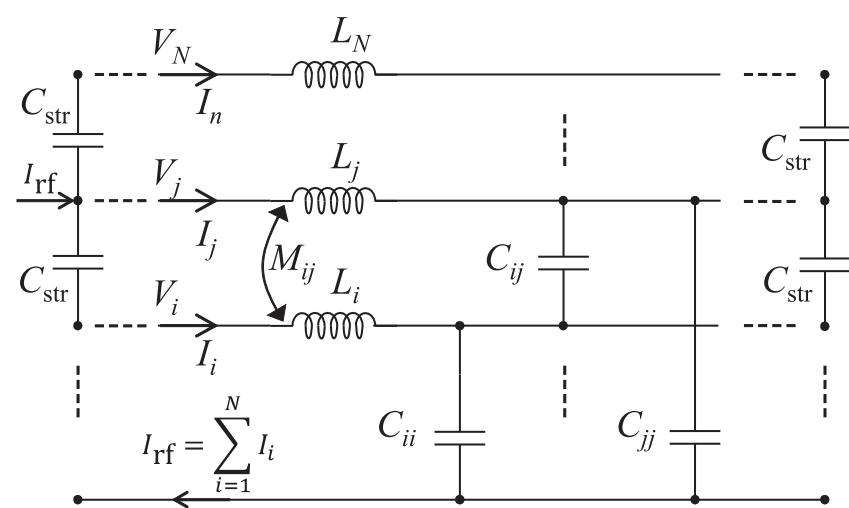

$0 \mathrm{~V}$ ground reference plane

Figure 2. The antenna equivalent circuit comprising a multi-leg transmission line terminated at both ends by stringer capacitors $C_{\text {str }}$. The $C_{i j}$ represent the per-unit-length capacitive coupling, and $M_{i j}$ the per-unit-length mutual partial inductance, between all legs, including their images induced in the plasma and in the reactor housing [31]. The reactor housing is the ground reference plane carrying the total RF return current. Only a few elements are shown for clarity.

voltages, for any arrangement of RF power feeding and ground connections (or electrically floating).

For the MTL model, referring to figure 2, the line voltages on the $N$ lines, with respect to the ground reference conductor, can be written as a column vector:

$$
\vec{V}(z)=\left(\begin{array}{c}
V_{1}(z) \\
V_{2}(z) \\
\vdots \\
V_{N}(z)
\end{array}\right)
$$

and similarly for the currents. The theoretical basis for the analysis of MTLs is given by Paul [24]. By analogy with single transmission line equations $[6,10,63]$, the generalized expressions for voltage and current are

$$
\begin{aligned}
& \mathrm{d} \vec{V}(z) / \mathrm{d} z=-\mathrm{j} \omega \bar{M} \vec{I}(z), \\
& \mathrm{d} \vec{I}(z) / \mathrm{d} z=-\mathrm{j} \omega \bar{C} \vec{V}(z),
\end{aligned}
$$

where $\bar{M}$ is the per-unit-length mutual partial inductance $N \times N$ matrix for the antenna legs [31], and $\bar{C}$ is the per-unitlength capacitance $N \times N$ matrix. In figure $2, M_{i j}$ is the mutual inductance between lines $i$ and $j$, and $L_{i} \equiv M_{i i}$ is the self-inductance of the $i$ th leg which is a special case of mutual inductance [64, 65]. The elements $M_{i j}$ and $C_{i j}$ of these matrices are generally complex, to account for the line series resistance and the dielectric parallel conductance, respectively. Mutual partial inductance exists between conductors which are parallel, but not between orthogonal conductors [65]. This means that one matrix can be defined for the legs, and another independently for the stringers; analytical expressions are given in [31]. The per-unit-length mutual partial inductance matrix for the legs, $\bar{M}$, is obtained by dividing by the leg length.

The transmission line is assumed to be uniform along $z$ so that $\bar{M}$ and $\bar{C}$ are constants. These two matrices are considered for the antenna, respectively without plasma and with plasma, in the next two sections. The uniform MTL wave

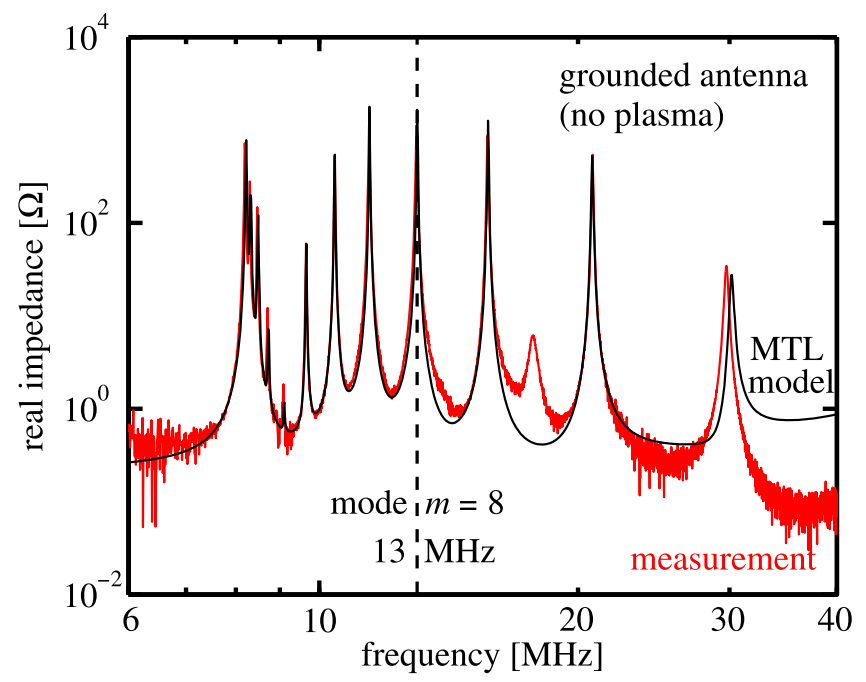

Figure 3. The real input impedance spectrum calculated using the MTL model compared with the network analyser measurement for a grounded antenna without plasma (RF feeding at A13; ground connections at A10 and A16). The $m=8$ mode is used in figures 4 and 7.

equation, analogous to the single transmission line [6, 10, 63], is:

$$
\mathrm{d}^{2} \vec{V}(z) / \mathrm{d} z^{2}=\left(-\omega^{2} \bar{M} \bar{C}\right) \vec{V}(z),
$$

for bi-symmetric matrices [24]. Also by analogy with single transmission line theory, the mode voltages have the solution [24]

$$
\vec{V}_{\mathrm{m}}(z)=\overline{\mathrm{e}^{-\Gamma} z} \vec{V}_{\mathrm{m}}^{+}+\overline{\mathrm{e}^{\mathrm{T}} z} \vec{V}_{\mathrm{m}}^{-},
$$

where $\bar{\Gamma}$ represents the complex propagation constants of the modes. The solution of the transmission line equations for the plasma-loaded antenna is given in appendix A.

\section{Experimental results and comparison with the MTL model for the antenna without plasma}

The MTL model was first verified by comparison with antenna measurements in air, without plasma. To access the antenna with voltage probes, these measurements were made with the top-plate removed as shown in figure 1(a). The $\bar{M}$ and $\bar{C}$ matrices then describe coupling between antenna legs and their images in the baseplate, for which wire-to-plane (microstrip) expressions are well known [24, 30, 31, 63, 65]. Either $\bar{M}$ or $\bar{C}$ can be calculated directly, and the other derived using $\bar{M} \bar{C}=\mu_{0} \epsilon_{0} \bar{I}$, where $\bar{I}$ is the identity matrix for this homogeneous dielectric (vacuum) [24].

\subsection{Measurements with a grounded antenna}

Comparison of the MTL calculation of antenna input impedance spectrum with the measurement shows very good agreement in figure 3. The lumped-element mutual inductance solution (not shown) [31] is not as accurate because the frequencies are over-estimated by about $1 \%$ when capacitive coupling is neglected. The influence of capacitive coupling is 

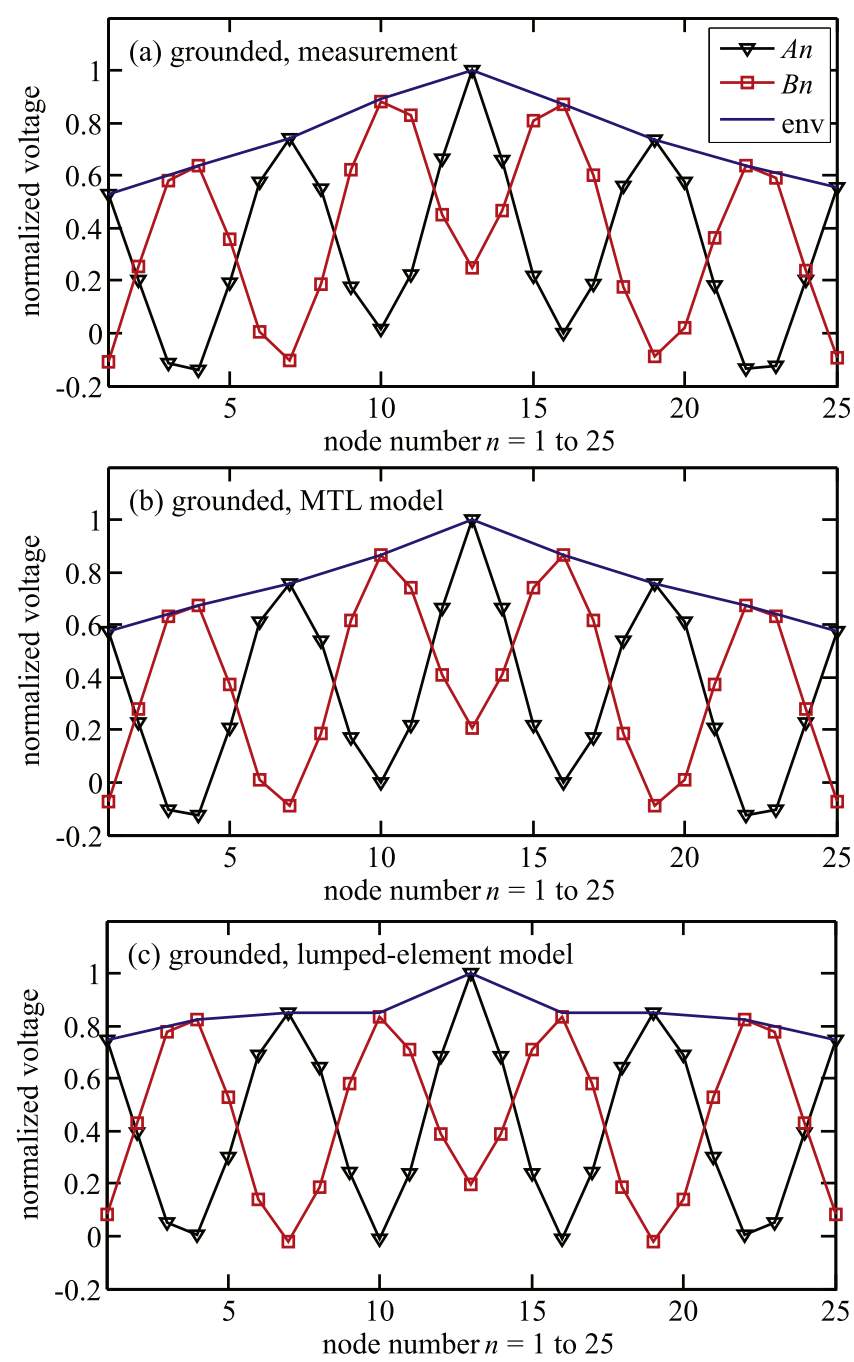

Figure 4. Comparison of the normalized node voltages $A n$ and $B n$ for $0.35 \mathrm{MHz}$ above the $13.0 \mathrm{MHz}$ resonance frequency of mode $m=8$ for the grounded antenna. (a) Measured with a voltage probe, and (b) calculated using the MTL model. (c) The same comparison, but calculated using a lumped-element mutual inductance model [31]. The lines 'env' show the amplitude envelope. Same grounding configuration as for figure 3 .

small for this case without plasma because the antenna is comparatively distant from the baseplate. Therefore, although the lumped-element model may be sufficiently accurate for vacuum calculations, it will be shown to be inadequate for plasma operation.

Comparison of the node voltage measurements $A n$ and $B n$ in figure 4(a) with the MTL solution in figure 4(b) also shows very good agreement, where the distortion of the voltage distribution due to off-resonance conditions is reproduced in detail. The amplitude envelope given by the lumped-element inductive approach [31] in figure 4(c) is clearly not as accurate, because the effects of capacitive coupling are neglected.

The antenna parameters are now fixed and no case-tocase adjustment is applied to the reactor equivalent circuit.

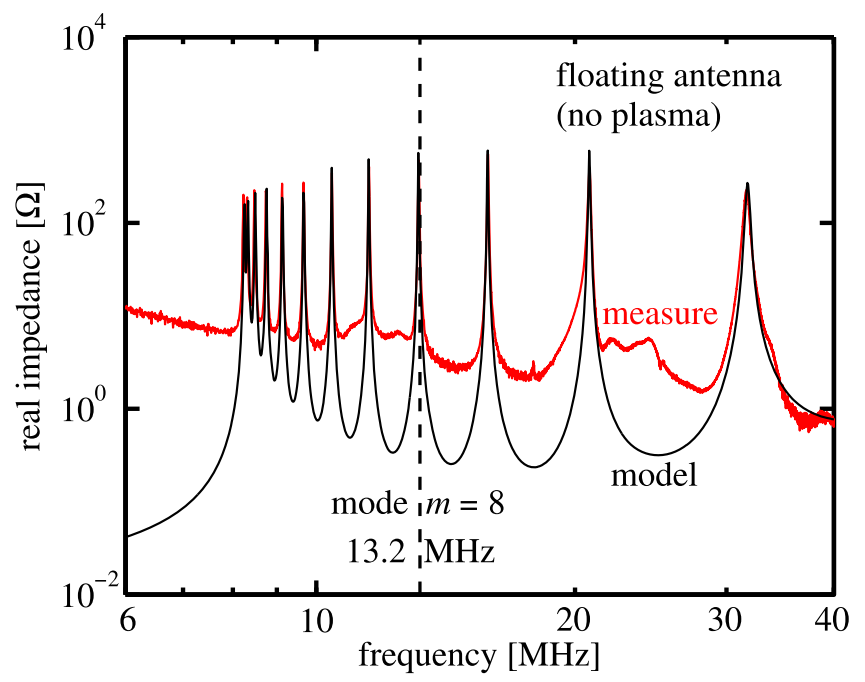

Figure 5. Real input impedance spectrum calculated using the MTL model compared with network analyser measurement for an electrically floating antenna without plasma (RF feeding at A13; no ground connections). The mode $m=8$ is used in figure 8 for measurements with plasma coupling.

\subsection{Measurements with an electrically floating antenna}

The MTL solution also provides very good agreement with the floating antenna input impedance spectrum measurement in figure 5. In this case, the ground return connections A10 and A16 in figure 1(b) were removed and measurements were performed with the antenna electrically floating (DC decoupled) with respect to ground. In the MTL model, the impedances of the A10 and A16 connectors were set to a very high value. The previous lumped-element inductive model [31] has no corresponding solution because capacitive coupling was not considered there. The measured and calculated node voltages (not shown) again agree in fine detail.

This good agreement with experimental results lends confidence to the MTL model for capacitive and inductive coupling between the antenna elements without plasma. In the following sections, this approach is extended to include antenna-plasma coupling, where the antenna input impedance effects are more clearly manifested because of the close proximity of the plasma to the antenna.

\section{Experimental and MTL model results for plasma coupling to the antenna}

For plasma experiments, the dielectric foam and glass window were installed and the reactor was closed with the topplate as shown in figure 1(b). The vacuum impedance measurements now correspond to a stripline because the antenna conductors are enclosed by both the baseplate and top-plate ground planes. Homogeneous stripline expressions for $\bar{M}$ and $\bar{C}$ are well known $[24,66,67]$. In presence of plasma, however, the situation is more complicated and the next two sections discuss how $\bar{M}$ and $\bar{C}$ can be estimated. 
grounded top-plate

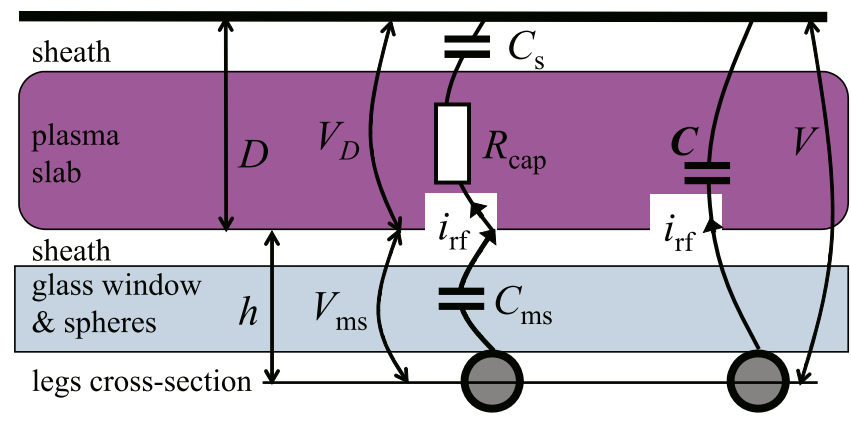

Figure 6. Schematic of capacitive coupling for the antenna legs to the plasma and top-plate, showing two equivalent circuits of the leg capacitances to ground. Capacitively coupled plasma dissipation is represented by the resistance $R_{\text {cap }}$

\subsection{Matrix of partial inductances $\bar{M}$ for the multi-conductor plasma-coupled antenna}

The tangential magnetic field due to the leg currents decays with the characteristic skin depth [63] as it penetrates the plasma, inducing a skin depth current. The complex image method was introduced previously to estimate the mutual inductive coupling between the antenna elements and their image current in a semi-infinite plasma slab [30,31]. The use of a complex skin depth $\mathbf{p}$ in the plasma accounts for both inductive coupling and ohmic dissipation in the antenna.

The complex image method is modified here to account for coupling with a resistive slab whose opposite boundary is limited by a conducting plate, as shown by Weisshaar et al [68]: in the expressions for mutual partial inductance [31], the complex distance from source to mirror plane [30], $h+\mathbf{p}$, is replaced by the effective complex distance $\mathbf{p}_{\mathrm{w}}=h+\mathbf{p} \tanh (D / \mathbf{p})$, where $D$ is the distance across the plasma to the top-plate (see figure 6). This expression selfconsistently accounts for image currents induced by the antenna in both the plasma and the top-plate.

Using the principle of superposition for magnetic flux, the net mutual partial inductance $\bar{M}$ of the antenna is the algebraic sum of the mutual partial inductance with all induced currents, including image currents in the baseplate as well as the plasma and top-plate [31]. $\bar{M}$ is a bi-symmetric Toeplitz matrix and so only one row or column needs to be calculated to define the whole matrix, thus reducing computation time.

\subsection{Capacitance matrix $\bar{C}$ for the multi-conductor plasma- coupled antenna}

The importance of capacitive coupling is demonstrated by the observation that plasma is maintained even when the antenna ground return connections, A10 and A16 in figure 1(b), are removed. The antenna is then electrically floating and it behaves as a CCP with an array of high voltage legs instead of a RF electrode plate. In this case, the RF feed current returns uniquely by capacitive coupling, principally via the plasma to the grounded top-plate. This is different from other ICP antennas which have ground connections, or at least a capacitor termination $[14,52,55]$. It is necessary to estimate the capacitance matrix between the antenna legs and the plasma.

The normal electric field in the sheath does not penetrate the plasma because of Debye screening [18], therefore the antenna-plasma system can be treated as a microstrip transmission line with conducting boundaries defined by the antenna legs and the adjacent plasma surface. The microstrip is assumed uniform along the legs, but it is inhomogeneous in the transverse plane because of the vacuum and the glass dielectric regions shown in figure 6.

The per-unit-length capacitance matrix of this inhomogeneous microstrip, $\overline{\bar{C}}_{\mathrm{ms}}$, was calculated from the inverse of Maxwell's potential coefficient matrix $\bar{P}[24,69]$ using the partial image method of Silvester [24, 67]. $\bar{P}$ is the matrix of induced potentials at every leg position for unit charge separately on each leg. It is also a bi-symmetric Toeplitz matrix and so $\bar{C}_{\mathrm{ms}}=\bar{P}^{-1}$ is bi-symmetric; it contains only real elements because the glass in figure 6 is effectively lossless.

A particularity of the microstrip here is that, although the plasma surface is the mirror plane for induced charge, it is not the ground plane; the ground plane is the top-plate on the far side of the plasma. With reference to figure 6 , the transverse RF currents are given by $\vec{i}_{\mathrm{rf}}=\mathrm{j} \omega \bar{C}_{\mathrm{ms}} \vec{V}_{\mathrm{ms}}$, where $\vec{V}_{\mathrm{ms}}$ is the microstrip voltage array between the antenna legs and the plasma surface. Respecting current continuity, as shown schematically in figure 6 , this can also be written in terms of the MTL parameters as $\vec{i}_{\mathrm{rf}}=\mathrm{j} \omega \bar{C} \vec{V}$, where $\vec{V}=\vec{V}_{\mathrm{ms}}+\vec{V}_{D}$ is the voltage array between the antenna and ground, and $\bar{C}$ is the required plasma-coupled capacitance matrix. Hence $\bar{C}=\bar{C}_{\mathrm{ms}} /\left(1+\vec{V}_{D} / \vec{V}_{\mathrm{ms}}\right)$.

Finally, using the simplified plasma equivalent circuit in figure 6, $\vec{V}_{D} / \vec{V}_{\mathrm{ms}} \sim \mathrm{j} \omega C_{\mathrm{ms}} R_{\text {cap }}$, for $C_{\mathrm{ms}} \ll C_{\mathrm{s}}$, where $C_{\mathrm{s}}$ is the sheath capacitance, and $R_{\text {cap }}$ is the capacitive coupling resistance accounting for ohmic and stochastic heating of electrons [14]. This means that the MTL capacitance matrix $\bar{C}$ has complex elements, which represent an effective conductivity and capacitively coupled power loss. It is difficult to estimate $R_{\text {cap }}$ [14] although it is expected that the plasma resistance is small compared to the capacitive impedance. In practice, $\vec{V}_{D} / \vec{V}_{\mathrm{ms}} \sim 0.1 \mathrm{j}$ gives a reasonable fit to the experimental data shown below. In view of these approximations, it would be superfluous and cumbersome to calculate the capacitance matrix for the complete experimental configuration, including multiple reflections of the antenna between the plasma and the distant baseplate in presence of the inhomogeneous dielectric. Instead, the capacitance matrix described above serves as a sufficient approximate example.

\subsection{Plasma measurements with a grounded antenna}

Figure 7(a) shows the grounded antenna input impedance spectra measured, with plasma, at the vacuum feedthrough for various RF powers using a variable frequency RF generator.

The observed shifts to frequencies below the vacuum resonance frequency are a clear indication of capacitive coupling of the antenna to the plasma. Figure 7(b) shows calculations of the corresponding input impedance mode 

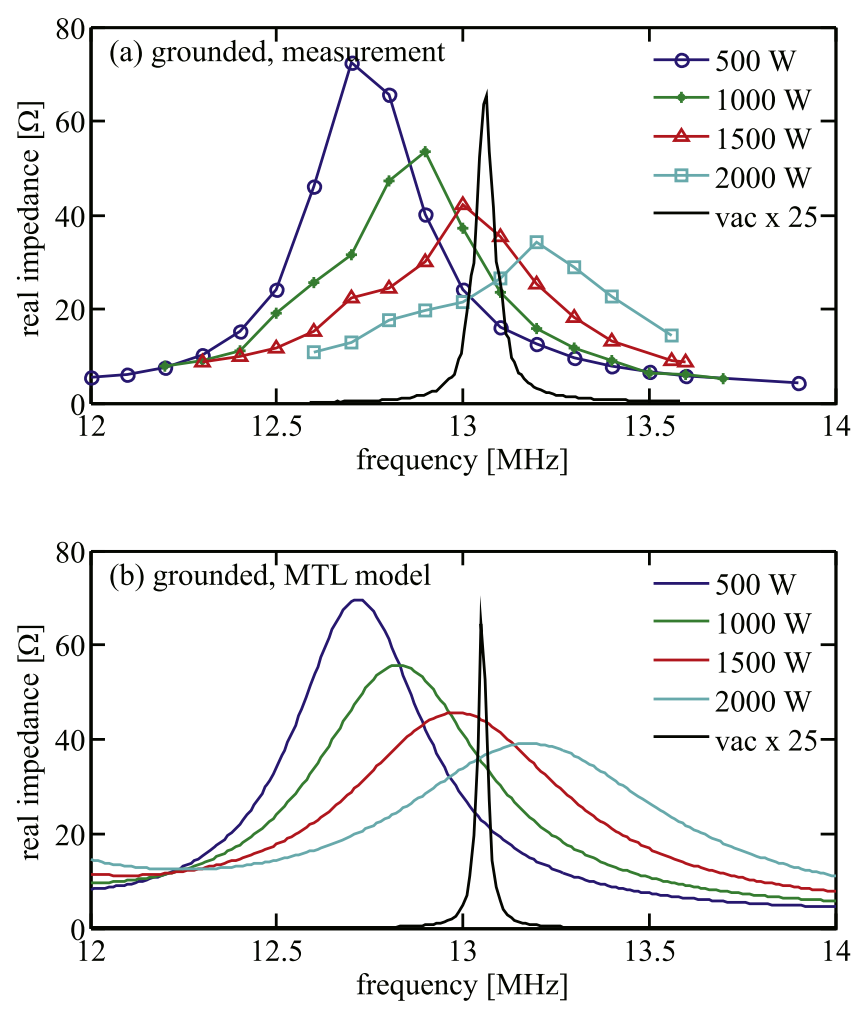

Figure 7. (a) Measurements of the mode $m=8$ impedance spectra for a grounded antenna in vacuum and with plasma for 500, 1000 , 1500 and $2000 \mathrm{~W}$ RF power. (b) Calculations of the same spectra using the multi-conductor transmission line model. Argon pressure $2 \mathrm{~Pa}$, same grounding configuration as for figure 3 .

spectra using the MTL model, which show reasonably good agreement for the frequency shifts and the strong reductions in input impedance in the presence of plasma loading of the antenna. Conversely, for purely inductive coupling [31], the mode resonance frequency would only increase above the vacuum resonance, and the lumped-element model results (not shown) over-estimate the mode frequencies by $\sim 0.7 \mathrm{MHz}(\sim 5 \%)$ and the mode impedance by a factor $\sim 2$. This is a large error compared to the corresponding case without plasma (section 4.1) because of the close proximity of the antenna, and therefore strong capacitive coupling, to the plasma.

The mutual inductive coupling of the plasma with the antenna in figure 7(b) was calculated using the complex image method $[30,31]$ according to section 5.1. The mutual inductance depends on geometrical dimensions and the plasma complex skin depth, given by the electron density and the electron-neutral collision frequency [30], as follows:

(i) The distance between the antenna leg axes and the plasma/sheath boundary, $h=1.05 \mathrm{~cm}$, was chosen according to $1 \mathrm{~cm}$ from the leg axes to the glass top surface, plus an estimated $0.3-0.5 \mathrm{~cm}$ for the sheath width (corresponding to about four Debye lengths for the undriven sheath in these plasma conditions [18]).

(ii) The argon pressure determines the electron-neutral collision frequency $\nu_{\mathrm{m}}\left[\mathrm{s}^{-1}\right] \approx p[\mathrm{~Pa}] \times 2.6 \times 10^{7}$ for typical electron temperature in these plasma conditions [18].

(iii) The plasma density was measured by Langmuir probe to be approximately $n_{\mathrm{e}} \approx\{0.8,1.2,1.7,2.4\} \times 10^{16} \mathrm{~m}^{-3}$ for delivered $\mathrm{RF}$ power $P_{\mathrm{rf}}=\{500,1000,1500,2000\} \mathrm{W}$.

These three parameters suffice for the complex image calculations [31] in figure 7(b).

The calculated range of real skin depth [31] goes from $7 \mathrm{~cm}($ at $500 \mathrm{~W}$ ) to $4 \mathrm{~cm}$ (at $2000 \mathrm{~W}$ ). For the lowest power, the associated skin depth is approximately equal to the electrode gap, and capacitive coupling could be expected to dominate because significant current is induced in the topplate instead of in the plasma. On the other hand, for the highest power, the skin depth is approximately half of the electrode gap and inductive coupling could be expected to dominate $[14,18,31]$. This is consistent with the frequency shift from negative (dominant capacitive coupling) to positive (dominant inductive coupling) in figure 7.

The RF power inductively coupled to the plasma via the mutual inductance is called the $H$-mode power transfer from the antenna ICP source, and the RF power coupled to the plasma by capacitive coupling is called the $E$-mode power transfer $[8,9,14]$. For the EMCP of these antennas, the transition from $E$-mode to $H$-mode can be indistinct [19], because both $E$ - and $H$-modes co-exist to some degree $[8,9,14,70]$.

\subsection{Plasma measurements with an electrically floating antenna}

For the floating antenna, the MTL model results in figure 8(b) are in fair agreement with the measurements in figure 8(a). The measured plasma densities in this case were $n_{\mathrm{e}} \approx\{0.7,1.7,2.1\} \times 10^{16} \mathrm{~m}^{-3}$ for delivered RF power $P_{\text {rf }}=\{500,1500,2000\} \mathrm{W}$.

The purely inductive coupling (lumped-element) model [31] over-estimates the mode frequencies by $\sim 0.48 \mathrm{MHz}$ ( $\sim 3.6 \%$ ) and the mode impedance by less than $20 \%$. This is a smaller error compared to the grounded case, possibly because capacitive currents to the plasma are weaker for the floating antenna.

\subsection{Plasma uniformity}

Uniformity is a key criterion for large area plasma processing. For this antenna, Langmuir probe density measurements in figure 9(a) typically show a flattened dome profile with approximately $50 \%$ drop in plasma density from the center to the antenna edges ${ }^{3}$. This plasma non-uniformity does not fulfil the condition of transmission line uniformity in section 3.1, although averaging over the whole antenna nevertheless gives reasonable agreement with the measured plasma-loaded antenna input impedance in figure 8 .

\footnotetext{
3 For these high power $(15 \mathrm{~kW})$ tests, $100 \mathrm{pF}$ was added to each stringer capacitor so that the mode resonance with plasma corresponded approximately to the industrial frequency $13.56 \mathrm{MHz}$. This correction was necessary because the initial design did not account for capacitive coupling.
} 

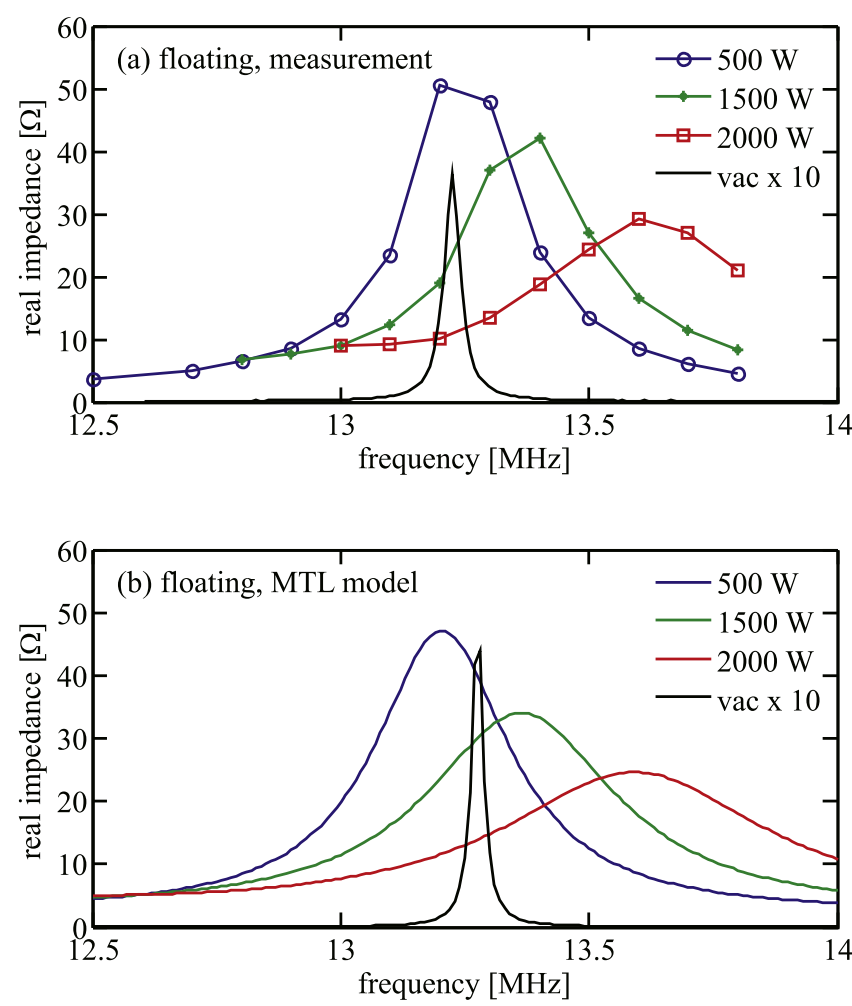

Figure 8. (a) Measurements of the mode $m=8$ impedance spectra for a floating antenna in vacuum and with plasma for 500, 1000 and 2000 W RF power. (b) Calculations of the same spectra using the multi-conductor transmission line model. Argon pressure 1.5 Pa, same floating configuration as for figure 5 .

In CCP reactors, $[15,71,72]$, the RF current circulates as a spatially distributed surface current in the skin depth of metal plate electrodes, whereas the current in ICP reactors circulates in discrete coils or antennas. For ICPs, the antenna pattern of these currents may appear on the substrate if the radical diffusion path is not long enough, requiring low pressure and wide electrode gap [12, 73]. However, the peaks in figure 9 (b) are due to the $m=8$ mode structure of the resonant antenna, not the individual legs. Note that low pressure ICP operation brings the advantage of no powder formation in deposition plasmas [29].

Figure 9(c) shows the measured variation in ion density along the length of the central leg. Using the MTL model, the calculated standing-wave variation of current and voltage along the legs is between $9 \%$ and $21 \%$, depending on whether the node position is near the middle or the end of a leg. This corresponds to a wavelength reduction factor $[15,62,74]$ of approximately 2.25 compared with the vacuum wavelength, due to the relative permittivity of the antenna-plasma system. The relative permittivity is a centro-symmetric matrix given by $\bar{M} \bar{C} /\left(\mu_{0} \epsilon_{0}\right)$; it is not a diagonal matrix because of the inhomogeneous dielectric-plasma system [24] and the different plasma penetration depths of the tangential magnetic and normal electric fields. However, the size and frequency of this reactor are not high enough to unambiguously demonstrate a standing wave non-uniformity in figure 9(c) because of the convolution with the diffusion profile $[75,76]$.
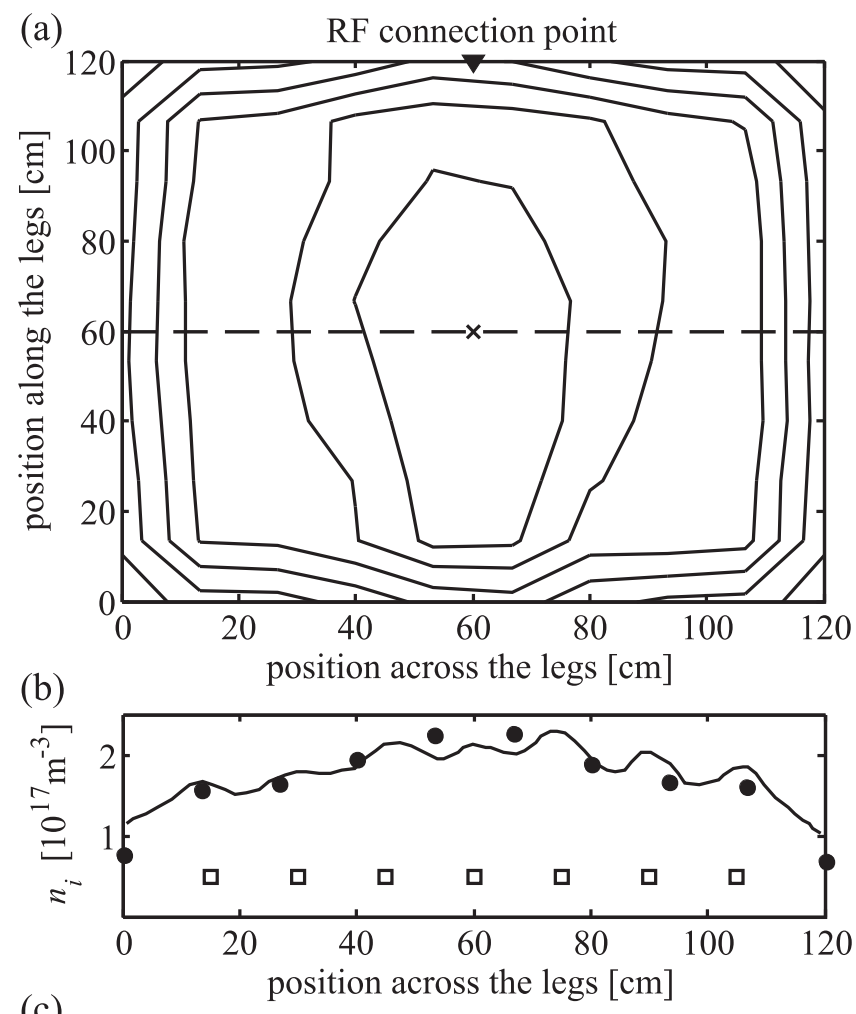

(c)

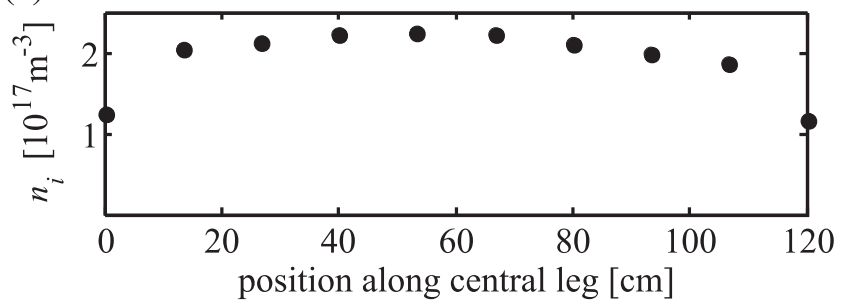

Figure 9. (a) Contour plot of the ion density $n_{\mathrm{i}}$ over the antenna area using a $10 \times 10$ array of surface probes. Contour levels descend successively by $\frac{1}{8}$ th of the central maximum of $2.3 \times 10^{17} \mathrm{~m}^{-3}$. (b) Measured density profile along the dashed line in (a), using a traversing Langmuir probe. The circles mark the surface probe measurements; the square symbols indicate the position of $m=8$ mode maxima which also correspond to figure 4. (c) Measured density profile along the central leg. Argon pressure $1.5 \mathrm{~Pa}, 15 \mathrm{~kW}$ input power at $13.56 \mathrm{MHz}$. Same floating configuration as for figure 5 .

\section{Conclusions}

A large antenna generates a plasma which is simultaneously a CCP and an ICP; it would therefore be better described as an electromagnetically coupled plasma (EMCP) source. A multiconductor transmission line (MTL) model is proposed to describe electromagnetic coupling in a large area planar ICP antenna. The inductive antenna-plasma coupling was calculated in terms of the per-unit-length mutual partial inductance matrix, using the complex image method $[30,31]$. The perunit-length capacitance matrix was estimated by analogy of capacitive coupling of the antenna to a plasma slab. The MTL matrix equations were solved by implementing the termination impedances of the resonant network. 
The MTL model reproduces the measurements of antenna input impedance spectra, with and without plasma coupling, for grounded and electrically floating antennas. The distinguishing feature of the model is that the mutual inductances and capacitive coupling between all elements of the antenna, plasma, and reactor are taken into account.

The model is valid for the whole range of skin depths in the plasma from much shorter to much longer than the reactor transverse dimensions. The MTL method is also valid for reactor lengths from shorter to longer than the effective wavelength of RF excitation in the plasma-coupled system. The model is analytic, with computation required only for the matrix solution.

The MTL approach could be applicable to model and design other planar ICP antenna sources (ladder, U-type, serpentine, double comb, etc) by applying the termination impedances appropriate to each antenna type.

\section{Acknowledgments}

We thank Dr Ch Hollenstein for many helpful comments, and Drs J Larrieu and P Fayet of Tetra Pak SA, Romont, Switzerland, for their advice and support. The development of the plasma source was supported by the Swiss Commission for Technology and Innovation grant no. CTI 14693.1 PFIW-IW.

\section{Appendix A. Solution of the MTL equations for the ICP antenna source}

\section{A.1. General solution of the MTL equations}

The uniform MTL wave equation, analogous to the single transmission line [6, 10, 63], is:

$$
\mathrm{d}^{2} \vec{V}(z) / \mathrm{d} z^{2}=\left(-\omega^{2} \bar{M} \bar{C}\right) \vec{V}(z)
$$

and identically for $\vec{I}(z)$, where the implicit commutability $\bar{M} \bar{C}=\bar{C} \bar{M}$ is assured by the matrix bi-symmetries [24] mentioned in sections 5.1 and 5.2. The equations can be decoupled by a similarity transformation [24]

$$
\vec{V}(z)=\bar{T}_{V} \vec{V}_{\mathrm{m}}(z)
$$

where $\bar{T}_{V}$ is the matrix whose $N$ columns are the eigenvectors of $\left(-\omega^{2} \bar{M} \bar{C}\right)$ and $\vec{V}_{\mathrm{m}}(z)$ is the array of mode voltages. The $N$ decoupled wave equations for the mode voltage amplitudes are

$$
\mathrm{d}^{2} \vec{V}_{\mathrm{m}}(z) / \mathrm{d} z^{2}=\bar{\Gamma}^{2} \vec{V}_{\mathrm{m}}(z),
$$

where $\bar{\Gamma}^{2}=\bar{T}_{V}^{-1}\left(-\omega^{2} \bar{M} \bar{C}\right) \bar{T}_{V}$ is the $N \times N$ diagonal matrix of propagation constant eigenvalues. By analogy with single transmission lines $[6,10,63]$, the $N$ mode voltages and currents have the solution [24]

$$
\begin{aligned}
& \vec{V}_{\mathrm{m}}(z)=\overline{\mathrm{e}^{-\Gamma} z} \vec{V}_{\mathrm{m}}^{+}+\overline{\mathrm{e}^{\Gamma} z} \vec{V}_{\mathrm{m}}^{-}, \\
& \vec{I}_{\mathrm{m}}(z)=\bar{Y}_{\mathrm{c}}\left(\overline{\mathrm{e}^{-\Gamma} z} \vec{V}_{\mathrm{m}}^{+}-\overline{\mathrm{e}^{\Gamma} z} \vec{V}_{\mathrm{m}}^{-}\right),
\end{aligned}
$$

where the matrix exponentials are defined as

$$
\overline{\mathrm{e}^{ \pm \Gamma z}}=\left(\begin{array}{cccc}
\mathrm{e}^{ \pm \Gamma_{1} z} & 0 & \cdots & 0 \\
0 & \mathrm{e}^{ \pm \Gamma_{2} z} & \ddots & \vdots \\
\vdots & \ddots & \ddots & 0 \\
0 & \cdots & 0 & \mathrm{e}^{ \pm \Gamma_{N} z}
\end{array}\right) .
$$

Here, $\vec{V}_{\mathrm{m}}^{+}$and $\vec{V}_{\mathrm{m}}^{-}$are $N \times 1$ arrays of complex phasor amplitudes of forward and backward waves, to be determined by boundary conditions, and $\bar{Y}_{\mathrm{c}}=(\mathrm{j} \omega \bar{M})^{-1} \bar{T}_{V} \bar{\Gamma} \bar{T}_{V}^{-1}$ is the characteristic admittance matrix of the line which depends on plasma coupling.

\section{A.2. Solution for the ICP resonant network antenna source}

The termination impedances appropriate to the resonant network shown in figures 1 and 2 are applied here. With regard to the appendix in [31], instead of using constant current and Ohm's law in the antenna legs, the voltage and current of each leg are now related by the transmission line equations. The solution consists in finding the values of the phasor amplitude vectors $\vec{V}_{\mathrm{m}}^{+}$and $\vec{V}_{\mathrm{m}}^{-}$for the $N$ legs. The lumped-element model for purely inductive coupling in [31] is recovered when capacitive coupling is neglected, i.e. in the limit $\bar{C} \rightarrow \overline{0}$.

Taking the origin for $z$ at the middle of the leg length $2 H$, the voltages $\vec{V}(z)$ and currents $\vec{I}(z)$ at both ends of the legs are $\vec{A}=\vec{V}(H), \vec{B}=\vec{V}(-H), \quad \overrightarrow{I_{A}}=\vec{I}(H), \quad \overrightarrow{I_{B}}=\vec{I}(-H)$. These voltages and currents are determined by the termination impedances in the stringers: using the terminology of [31], Ohm's law for the stringers gives

$$
\begin{aligned}
\bar{U}_{T} \vec{A} & =\bar{Z}^{\text {line } \vec{J}}+\bar{Z}^{\text {opp } \vec{K},} \\
\bar{U}_{T} \vec{B} & =\bar{Z}^{\text {line }} \vec{K}+\bar{Z}^{\text {opp } \vec{J},}
\end{aligned}
$$

where $\vec{J}, \vec{K}$ are the currents in each line of stringers, $\bar{Z}^{\text {line }}$ is the impedance matrix of stringers, including mutual inductance to all other in-line stringers, and $\bar{Z}^{\text {opp }}$ is the mutual inductance matrix with all opposing stringers. For the resonant network in this paper, $\bar{Z}^{\text {line }}$ includes the stringer capacitors $C_{\text {str }}$, but other ICP linear array types can be modeled using termination impedances appropriate to the stringers of each particular antenna. Current conservation at the nodes [31] requires

$$
\begin{aligned}
\bar{U} \vec{J} & =\overrightarrow{I_{A}}+\overrightarrow{S_{A}}, \\
\bar{U} \vec{K} & =-\overrightarrow{I_{B}}+\overrightarrow{S_{B}},
\end{aligned}
$$

where $\vec{S}_{A, B}$ are the source current vectors. A relation between the node voltages and currents can be found by eliminating $\vec{J}$, $\vec{K}$ to obtain:

$$
\begin{aligned}
& \vec{A}=\overline{m_{1}}\left(\overrightarrow{I_{A}}+\overrightarrow{S_{A}}\right)-\overline{m_{2}}\left(\overrightarrow{I_{B}}-\overrightarrow{S_{B}}\right), \\
& \vec{B}=\overline{m_{2}}\left(\overrightarrow{I_{A}}+\overrightarrow{S_{A}}\right)-\overline{m_{1}}\left(\overrightarrow{I_{B}}-\overrightarrow{S_{B}}\right),
\end{aligned}
$$

where $\bar{m}_{1}=\bar{U}_{T}^{-1} \bar{Z}^{\text {line }} \bar{U}^{-1}$ and $\bar{m}_{2}=\bar{U}_{T}^{-1} \bar{Z}^{\text {opp }} \bar{U}^{-1}$. The solution is facilitated by defining

$$
\begin{aligned}
\bar{C}_{h} & =\overline{\mathrm{e}^{\Gamma} H}+\overline{\mathrm{e}^{-\Gamma} H}, \\
\bar{S}_{h} & =\overline{\mathrm{e}^{\Gamma} H}-\overline{\mathrm{e}^{-\Gamma} H},
\end{aligned}
$$


with a change of variables:

$$
\begin{aligned}
\vec{\Delta} & =\vec{V}_{\mathrm{m}}^{+}-\vec{V}_{\mathrm{m}}^{-}, \\
\vec{\sigma} & =\vec{V}_{\mathrm{m}}^{+}+\vec{V}_{\mathrm{m}}^{-} .
\end{aligned}
$$

With these definitions, according to (A.2) and (A.4)

$$
\begin{aligned}
\vec{A} & =\bar{T}_{V}\left(\bar{C}_{h} \vec{\sigma}-\bar{S}_{h} \vec{\Delta}\right) / 2, \\
\vec{B} & =\bar{T}_{V}\left(\bar{C}_{h} \vec{\sigma}+\bar{S}_{h} \vec{\Delta}\right) / 2, \\
\overrightarrow{I_{A}} & =\bar{Y}_{\mathrm{c}} \bar{T}_{V}\left(\bar{C}_{h} \vec{\Delta}-\bar{S}_{h} \vec{\sigma}\right) / 2, \\
\vec{I}_{B} & =\bar{Y}_{\mathrm{c}} \bar{T}_{V}\left(\bar{C}_{h} \vec{\Delta}+\bar{S}_{h} \vec{\sigma}\right) / 2 .
\end{aligned}
$$

Introducing these expressions into (A.8) for $\vec{B}-\vec{A}$ and $\vec{B}+\vec{A}$ respectively, gives

$$
\begin{aligned}
& {\left[\left(\bar{m}_{1}-\bar{m}_{2}\right)^{-1} \bar{T}_{V} \bar{S}_{h}+\bar{Y}_{\mathrm{c}} \bar{T}_{V} \bar{C}_{h}\right] \vec{\Delta}} \\
& \quad=\overrightarrow{S_{B}}-\overrightarrow{S_{A}}=\bar{O}_{D} \vec{\Delta}, \\
& {\left[\left(\bar{m}_{1}+\bar{m}_{2}\right)^{-1} \bar{T}_{V} \bar{C}_{h}+\bar{Y}_{\mathrm{c}} \bar{T}_{V} \bar{S}_{h}\right] \vec{\sigma}} \\
& \quad=\overrightarrow{S_{B}}+\overrightarrow{S_{A}}=\bar{O}_{S} \vec{\sigma},
\end{aligned}
$$

which define the matrices $\bar{O}_{D, S}$ as shorthand pre-factors of $\vec{\Delta}, \vec{\sigma}$ respectively. The source current vectors $\vec{S}_{A}$ and $\vec{S}_{B}$ must account for the imposed input currents as well as for possible connections to ground. Considering an input current $I_{\text {in }}$ injected into a single node $A_{\text {in }}$ (node A13 in figure 1), we set

$$
\begin{aligned}
& \vec{S}_{A}=\vec{I}_{\text {in }}-\vec{Y}_{A} \vec{A}, \\
& \vec{S}_{B}=-\vec{Y}_{B} \vec{B},
\end{aligned}
$$

where the vector $\vec{I}_{\text {in }}$ has zeros for all components except for $I_{\text {in }}$ at $A_{\text {in }}$, and $\vec{Y}_{A, B}$ are diagonal matrices with the ground connector admittance at the relevant indices (nodes A10 and A16 in figure 1 , with $\vec{Y}_{B}=0$ ). The expressions for $\overrightarrow{S_{B}} \pm \overrightarrow{S_{A}}$ in (A.12), which were derived from the network currents and voltages, can be compared with expressions (A.13) for the source currents by using (A.11) to write:

$$
\begin{aligned}
\overrightarrow{S_{B}}-\vec{S}_{A}= & \left(\vec{Y}_{A}-\vec{Y}_{B}\right) \bar{T}_{V} \bar{C}_{h} \vec{\sigma} / 2 \\
& -\left(\vec{Y}_{A}+\vec{Y}_{B}\right) \bar{T}_{V} \bar{S}_{h} \vec{\Delta} / 2-\vec{I}_{\text {in }} \\
= & \bar{O}_{1} \vec{\sigma}+\bar{O}_{2} \vec{\Delta}-\vec{I}_{\text {in }} \\
\vec{S}_{B}+\vec{S}_{A}= & -\left(\vec{Y}_{A}+\vec{Y}_{B}\right) \bar{T}_{V} \bar{C}_{h} \vec{\sigma} / 2 \\
& +\left(\vec{Y}_{A}-\vec{Y}_{B}\right) \bar{T}_{V} \bar{S}_{h} \vec{\Delta} / 2+\vec{I}_{\text {in }} \\
= & \bar{O}_{3} \vec{\sigma}+\bar{O}_{4} \vec{\Delta}+\vec{I}_{\text {in }},
\end{aligned}
$$

where the matrices $\bar{O}_{1-4}$ are shorthand pre-factors of $\vec{\Delta}, \vec{\sigma}$. Using the pre-factor simplifications, equating (A.12) and (A.14) gives the following simultaneous equations for $\vec{\Delta}$ and $\vec{\sigma}$

$$
\begin{aligned}
\left(\bar{O}_{D}-\bar{O}_{2}\right) \vec{\Delta}-\bar{O}_{1} \vec{\sigma} & =-\vec{I}_{\text {in }}, \\
\left(\bar{O}_{S}-\bar{O}_{3}\right) \vec{\sigma}-\bar{O}_{4} \vec{\Delta} & =+\vec{I}_{\text {in }},
\end{aligned}
$$

whose solution gives the required voltage solution using (A.10) and (A.2).

\section{References}

[1] d'Agostino R, Favia P, Kawai Y, Ikegami H, Sato N and Arefi-Khonsari F (ed) 2008 Advanced Plasma Technology. (Weinheim: Wiley)

[2] Kawai Y, Ikegami H, Sato N, Matsuda A, Uchino K, Kuzuya M and Mizuno A (ed) 2008 Industrial Plasma Technology (Weinheim: Wiley)

[3] Pizzini S 2012 Advanced Silicon Materials for Photovoltaic Applications (New York: Wiley)

[4] Kim S S, Chang H Y, Chang C S and Yoon N S 2000 Appl. Phys. Lett. 77492

[5] Jun H-S and Chang H-Y 2008 Appl. Phys. Lett. 92041501

[6] Lamm A J 1997 J. Vac. Sci. Technol. A 152615

[7] Khater M H and Overzet L J 2000 Plasma Sources Sci. Technol. 9545

[8] Sugai H, Nakamura K and Suzuki K 1994 Japan J. Appl. Phys. 332189

[9] Lee S-H, Cho J-H, Huh S-R and Kim G-H 2014 J. Phys. D: Appl. Phys. 47015205

[10] Wu Y and Lieberman M A 2000 Plasma Sources Sci. Technol. 9210

[11] Kushner M J, Collison W Z, Grapperhaus M J, Holland J P and Barnes M S 1996 J. Appl. Phys. 801337

[12] Park S E, Cho B U, Lee J K, Lee Y J and Yeom G Y 2003 IEEE Trans. Plasma. Sci. 31628

[13] Kawamura E, Graves D B and Lieberman M A 2011 Plasma Sources Sci. Technol. 20035009

[14] Chabert P and Braithwaite N S J 2011 Physics of RadioFrequency Plasmas (Cambridge: Cambridge University Press)

[15] Lieberman M A, Booth J P, Chabert P, Rax J M and Turner M M 2002 Plasma Sources Sci. Technol. 11283

[16] Sansonnens L, Howling A A and Hollenstein C 2006 Plasma Sources Sci. Technol. 15302

[17] Strobel C, Zimmermann T, Albert M, Bartha J W and Kuske J 2009 Sol. Energy Mater. Sol. Cells 931598

[18] Lieberman M A and Lichtenberg A J 2005 Principles of Plasma Discharges and Materials Processing 2nd edn (Hoboken, NJ: Wiley)

[19] Hollenstein C, Guittienne P and Howling A A 2013 Plasma Sources Sci. Technol. 22055021

[20] Piejak R B, Godyak V A and Alexandrovich B M 1992 Plasma Sources Sci. Technol. 1179

[21] Gudmundsson J T and Lieberman M A 1998 Plasma Sources Sci. Technol. 783

[22] Colpo P, Ernst R and Rossi F 1999 J. Appl. Phys. 851366

[23] Mashima H, Takeuchi Y, Noda M, Murata M, Naitou H, Kawasaki I and Kawai Y 2003 Surf. Coat. Technol. 171167

[24] Paul C R 2008 Analysis of Multiconductor Transmission Lines 2nd edn (Hoboken, NJ: Wiley)

[25] Guittienne P, Lecoultre S, Fayet P, Larrieu J, Howling A A and Hollenstein C 2012 J. Appl. Phys. 111083305

[26] Lecoultre S, Guittienne P, Howling A A, Fayet P and Hollenstein C 2012 J. Phys. D: Appl. Phys. 45082001

[27] Guittienne P, Fayet P, Larrieu J, Howling A A and Hollenstein C 2012 55th Annual SVC (Surface Vacuum Coaters) Technical Conf. (Santa Clara, CA, USA, 28 April3 May)

[28] Fayet P, Guittienne P, Howling A A and Larrieu J 2014 Whistler wave heated discharges in planar geometry 10th Int. Conf. Coatings on Glass and Plastics (Dresden, Germany)

[29] Demolon P, Guittienne P, Howling A A, Jost S and Furno I 2017 in preparation

[30] Howling A A, Guittienne P, Jacquier R and Furno I 2015 Plasma Sources Sci. Technol. 24065014 
[31] Guittienne P, Jacquier R, Howling A A and Furno I 2015 Plasma Sources Sci. Technol. 24065015

[32] Takeuchi Y, Nawata Y, Ogawa K, Serizawa A, Yamauchi Y and Murata M 2001 Thin Solid Films 386133

[33] Takeuchi Y, Kawasaki I, Mashima H, Murata M and Kawai Y 2001 Thin Solid Films 390217

[34] Takatsuka H, Noda M, Yonekura Y, Takeuchi Y and Yamauchi Y 2004 Sol. Energy 77951

[35] Takatsuka H, Yamauchi Y, Kawamura K, Mashima H and Takeuchi Y 2006 Thin Solid Films 506-507 13

[36] Nishimiya T, Takeuchi Y, Yamauchi Y, Takatsuka H, Kai Y, Muta H and Kawai Y 2007 Plasma Process. Polym. 4 S991

[37] Nishimiya T, Takeuchi Y, Yamauchi Y, Takatsuka H, Shioya T, Muta H and Kawai Y 2008 Thin Solid Films 5164430

[38] Yamauchi Y, Takeuchi Y, Takatsuka H, Yamashita H, Muta H and Kawai Y 2008 Contrib. Plasma Phys. 48326

[39] Murata M, Takeuchi Y, Sasagawa E and Hamamoto K 1996 Rev. Sci. Instrum. 671542

[40] Wendt A E and Mahoney L J 1996 Pure Appl. Chem. 681055

[41] Murata M, Mashima H, Yoshioka M, Nishida S, Morita S and Kawai Y 1997 Japan J. Appl. Phys. 364563

[42] Kawai Y, Yoshioka M, Yamane T, Takeuchi Y and Murata M 1999 Surf. Coat. Technol. 116-119 662

[43] Mashima H, Murata M, Takeuchi Y, Yamakoshi H, Horioka T, Yamane T and Kawai Y 1999 Japan J. Appl. Phys. 384305

[44] Kim K N, Lee Y J, Kyong S J and Yeom G Y 2004 Surf. Coat. Technol. 177-178 752

[45] Lim J H, Kim K N and Yeom G Y 2007 Plasma Process. Polym. 4 S 999

[46] Takagi T, Ueda M, Ito N, Watabe Y and Kondo M 2006 Japan J. Appl. Phys. 454003

[47] Takagi T, Ueda M, Ito N, Watabe Y, Sato H and Sawaya K 2006 Thin Solid Films $\mathbf{5 0 2} 50$

[48] Kim K N, Lee Y J, Jung S J and Yeom G Y 2004 Japan J. Appl. Phys. 434373

[49] Lim J H, Kim K N, Park J K, Lim J T and Yeom G Y 2008 Appl. Phys. Lett. 92051504

[50] Lim J H, Kim K N, Gweon G H, Park J B and Yeom G Y 2009 Pl. Chem. Pl. Proc. 29251

[51] Forgotson N, Khemka V and Hopwood J 1996 J. Vac. Sci. Technol. B 14732

[52] Suzuki K, Konishi K, Nakamura K and Sugai H 2000 Plasma Sources Sci. Technol. 9199

[53] Intrator T and Menard J 1996 Plasma Sources Sci. Technol. 5371
[54] Menard J and Intrator T 1996 Plasma Sources Sci. Technol. 5363

[55] Setsuhara Y, Miyake S, Sakawa Y and Shoji T 1999 Japan J. Appl. Phys. 384263

[56] Meziani T, Colpo P and Rossi F 2001 Plasma Sources Sci. Technol. 10276

[57] Colpo P, Meziani T and Rossi F 2005 J. Vac. Sci. Technol. A 23270

[58] Meziani T, Colpo P and Rossi F 2006 J. Appl. Phys. 99033303

[59] Yu Z, Shaw D, Gonzales P and Collins G J 1995 J. Vac. Sci. Technol. A 13871

[60] Advanced Energy Industries Inc www.advanced-energy.com.

[61] Jin J 1998 Electromagnetic Analysis and Design in Magnetic Resonance Imaging (Boca Raton, FL: CRC Press)

[62] Schmitt J P M, Elyaakoubi M and Sansonnens L 2002 Plasma Sources Sci. Technol. 11 A206

[63] Paul C R and Nasar S A 1987 Introduction to Electromagnetic Fields (New York: McGraw-Hill)

[64] Grover F W 1962 Inductance Calculations: Working Formulas and Tables (New York: Dover)

[65] Paul C R 2010 Inductance: Loop and Partial (Hoboken, NJ: Wiley)

[66] Kammler D W 1968 IEEE Trans. Microw. Theory Tech. MTT16925

[67] Silvester P 1968 Proc. IEE 11543

[68] Weisshaar A, Lan H and Luoh A 2002 IEEE Trans. Adv. Packag. 25288

[69] Harrington R F 1958 Introduction to Electromagnetic Engineering (New York: McGraw-Hill)

[70] Ahr P, Schüngel E, Schulze J, Tsankov T V and Czarnetzki U 2015 Plasma Sources Sci. Technol. 24044006

[71] Chabert P, Raimbault J-L, Levif P, Rax J-M and Lieberman M A 2006 Plasma Sources Sci. Technol. 15 S130

[72] Liu Y-X, Zhang Y-R, Bogaerts A and Wang Y-N 2016 J. Vac. Sci. Technol. A 33020801

[73] Kim D W, You S J, Kim J H, Chang H Y and Oh W Y 2015 IEEE Trans. Plasma. Sci. 433876

[74] Schmidt H, Sansonnens L, Howling A A, Hollenstein C, Elyaakoubi M and Schmitt J P M 2004 J. Appl. Phys. 954559

[75] Vahedi V, Lieberman M A, DiPeso G, Rognlien T D and Hewett D 1995 J. Appl. Phys. 781446

[76] Stittsworth J A and Wendt A E 1996 Plasma Sources Sci. Technol. 5429 